\title{
A study on some taxa of family Mniaceae (Bryophyta) in Darjeeling (West Bengal), India
}

\author{
Omar I, V Sahu* \& A K Asthana \\ Bryology Laboratory, CSIR-National Botanical Research Institute, Lucknow 226 001, India \\ *Email: sahuvinay8@gmail.com
}

\author{
ARTICLE HISTORY \\ Received: 29 October 2019 \\ Accepted: 03 February 2020 \\ Published: 01 April 2020

\section{KEYWORDS} \\ Mniaceae; Darjeeling; Mnium; \\ Plagiomnium; Orthomnion
}

\begin{abstract}
During study on the family Mniaceae in Darjeeling and its neighbouring areas, three genera and six species (Mnium lycopodioides, Orthomnion bryoides, Plagiomnium acutum, P. confertidens, $P$. rhynchophorum and $P$. succulentum) have been identified. Of these Plagiomnium acutum is reported here for the first time from eastern Himalaya. A detailed morpho-taxonomic account of these species with their current status and a key to all the taxa of family Mniaceae in Darjeeling is provided here.
\end{abstract}

\section{Introduction}

Darjeeling is located in West Bengal, surrounded by Sikkim in North, Bihar in South, Kalimpong in East and Nepal in West, at an elevation of $6700 \mathrm{ft}$. The humidity usually ranges between 72 to $96 \%$ and annual temperature ranges between 2 to $19^{\circ} \mathrm{C}$ with $309.2 \mathrm{~cm}$ annual precipitation. Darjeeling has a temperate climate and the vegetation comprises of Sal, oak, semi evergreen temperate and alpine forests. The members of family Mniaceae Schwägr. are distributed all over the world in temperate regions where they grow in moist and shady places on rocks and bark of trees. The family has been treated by several authors from time to time and only later, a reasonable criterion for demarcation of different genera has been developed (1). Family Mniaceae on worldwide basis contains nine genera and about 75 infra-generic taxa but in India five genera (Mnium Hedw., Orthomnion Wils., Plagiomnium T. J. Kop., Pseudobryum (Kindb.) T. J. Kop. and Rhizomnium T. J. Kop.) and about 27 taxa are present (2-7).

As far as diversity of family Mniaceae in Darjeeling is concerned, earlier researchers had documented 18 taxa $(4-6,8-12)$. Of these, 14 taxa are recognized except Mnium laevinerve Card. which is synonymised under $M$. lycopodioides Schwaegr., $M$. undulatum Hedw. is not found in India (6), $M$. marginatum (With.) P. Beauv. var. riparium and $M$. pseudopunctatum Bruch \& Schimp. earlier described now considered as Mnium lycopodioides and Rhizomnium nudum (Britt. \& Williams) T. J. Kop. respectively $(4,6,13)$. During the present study, out of these 14, six taxa have encountered including Plagiomnium acutum (Lindb.) T. J. Kop. which is recorded here for the first time from eastern Himalaya (4-7). The members of this family are remarkable for being almost confined to moist and shady places and having significant diagnostic characters like erect and prostrate habit, smooth or serrated leaf margin, teeth on leaves double or single or none, the anatomy of costa as well as border and pattern of leaf cells, pitted or not (1). At present, no up to date information is available on the diversity in family Mniaceae in India and hence a taxonomic revision is needed.

\section{Materials and Methods}

The present study is based upon the specimens collected from different areas of Darjeeling. The specimens have been deposited in the Bryophyte Herbarium, National Botanical Research Institute, Lucknow (LWG). Plants were soaked in water to regain their natural form and then identified with the help of binocular and Leica microscope. Plants were also photographed for easy identification of species.

(C) Omar et al. (2020). This is an open-access article distributed under the terms of the Creative Commons Attribution License, which permits unrestricted use, distribution, and reproduction in any medium, provided the original author and source are credited (https://creativecommons.org/licenses/by/4.0/).

To cite this article: Omar I, Sahu V, Asthana AK. A study on some taxa of family Mniaceae (Bryophyta) in Darjeeling (West Bengal), India. Plant Science Today. 2020;7(2):193-200. https://doi.org/10.14719/pst.2020.7.2.647 


\section{Results}

\section{Taxonomic treatment}

\section{Key to the taxa of family Mniaceae in Darjeeling}

1a. Leaves serrated, costa with stereids .2

1b. Leaves entire, costa without stereids 11 2a. Plants reddish, erect, without plagiotropic shoots, marginal teeth of leaf double (paired), costa with two stereid bands 3

2b. Plants green to brown, with plagiotropic shoots, marginal teeth of leaf single, costa with one stereid band
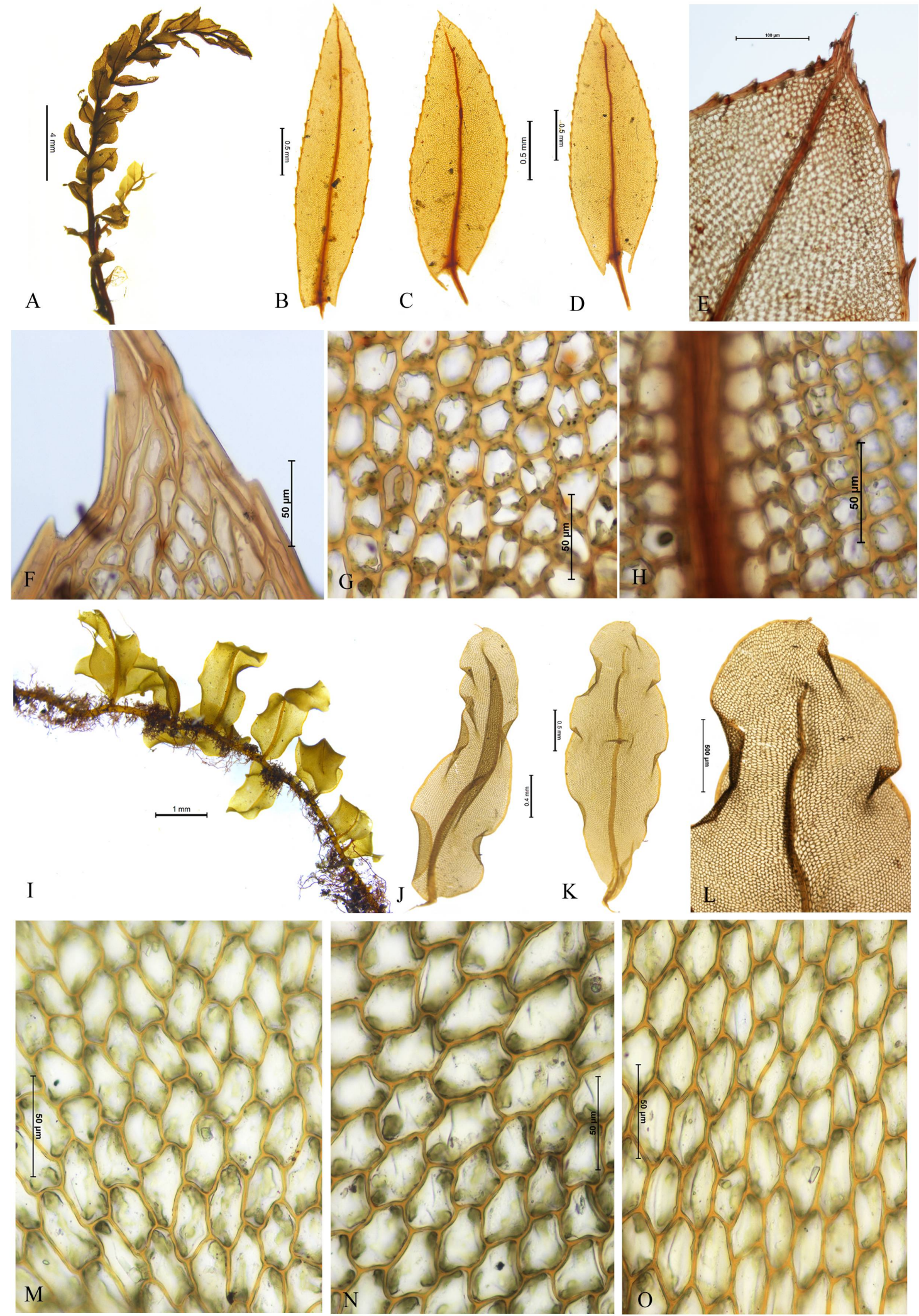

Fig.1. (A-O). Mnium lycopodioides Schwager. (LWG 225364C) A-H: A. Plant habit; B-D. Leaves; E. Leaf apex; F. Leaf apical cell; G. Leaf basal cells; H. Leaf median cells. Orthomnion bryoides (Griffith) Nork. (LWG 225495B) I-O: I. Plant habit; J, K. Leaves; L. Leaf apex; M. Leaf apical cells; $\mathbf{N}$. Leaf median cells; $\mathbf{O}$. Leaf basal cells. 

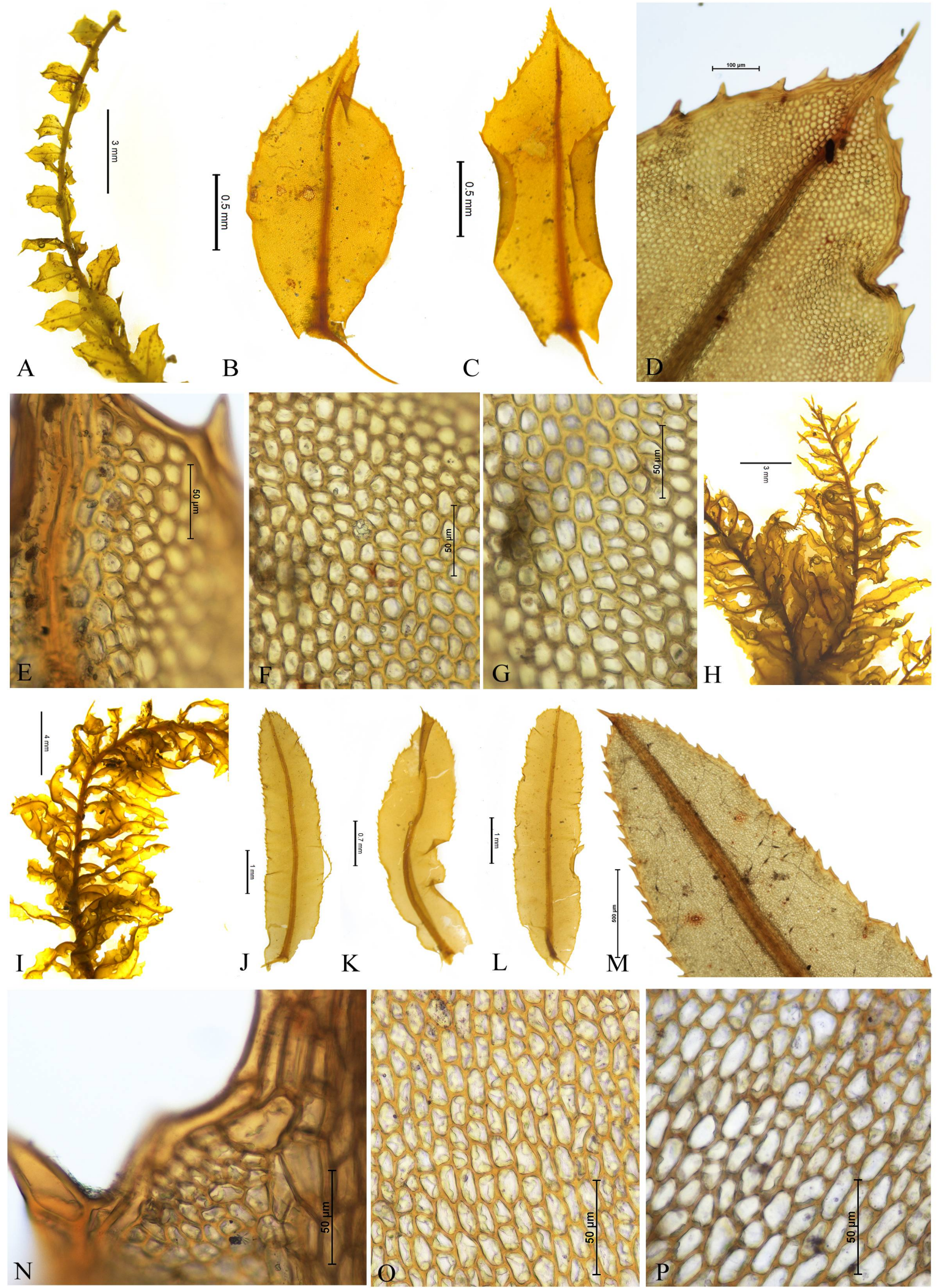

Fig. 2. (A-P). Plagiomnium acutum (Lindb.) T. J. Kop. (LWG 223977A) A-G: A. Plant habit; B, C. Leaves;; D. Leaf apex; E. Leaf apical cells; F. Leaf median cells; G. Leaf basal cells. Plagiomnium confertidens (Lindb. \& Arnell) T. J. Kop. (LWG 225364C); H-P: H, I. Plant habit; J-L. Leaves; M. Leaf apex; N. Leaf apical cells; O. Leaf median cells; P. Leaf basal cells.

3a. Leaves ovate-lanceolate to broadly elliptic, laminal cells heterogenous $(22.5-30 \mu \mathrm{m})$ Mnium lycopodiodes*

3b. Leaves elliptic to narrowly elliptic, laminal cells homogenous $\mu \mathrm{m})$

Mnium thomsonii 4a. Leaf middle.

margin

toothed Plagiomnium acutum*

4b. Leaf margin toothed up to base..

5a. Leaf marginal teeth long and sharp....................6

5b. Leaf marginal teeth small and blunt. .7 


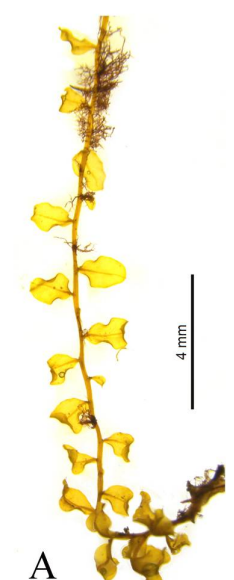

$$
\text { A }
$$
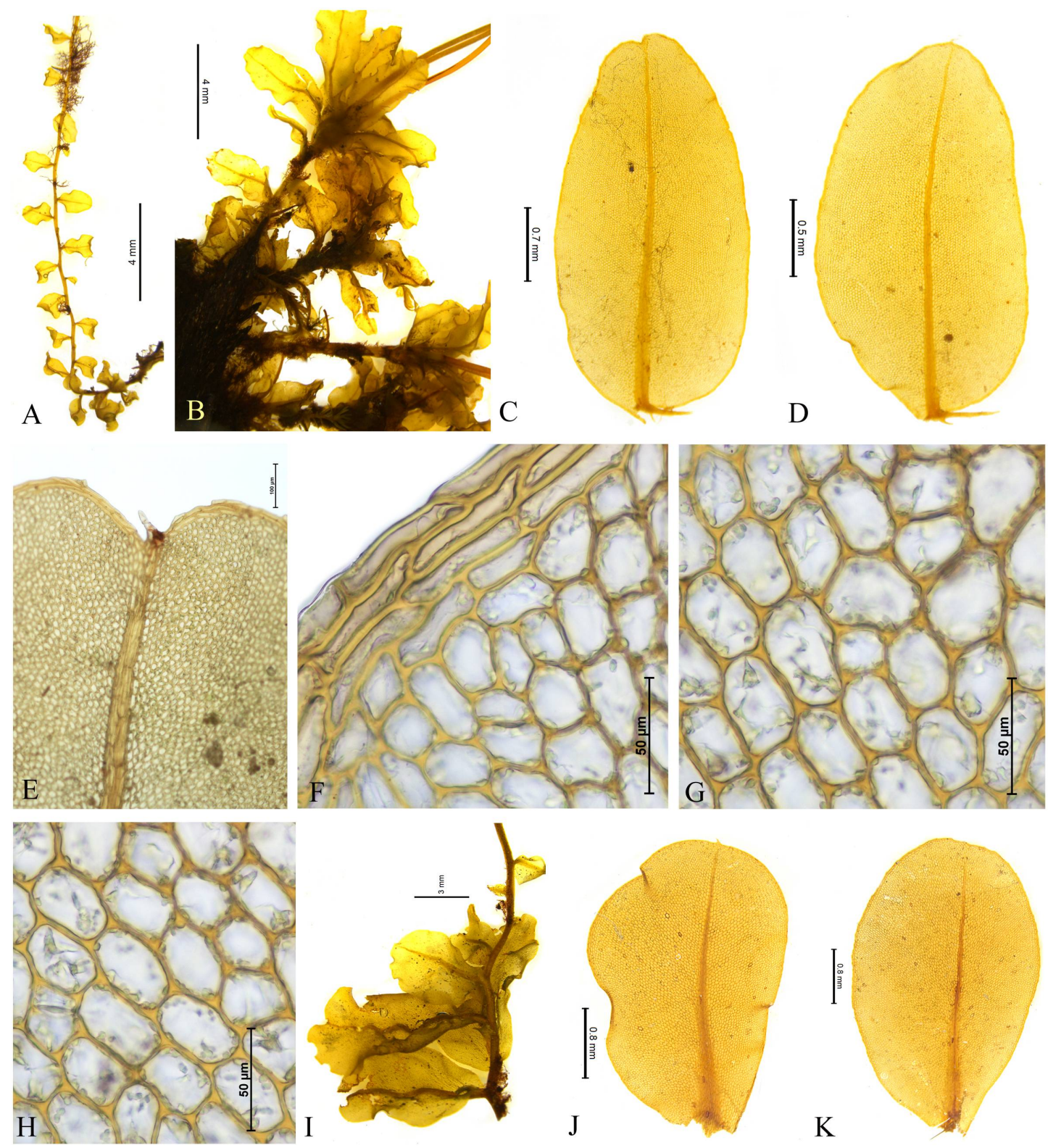

K
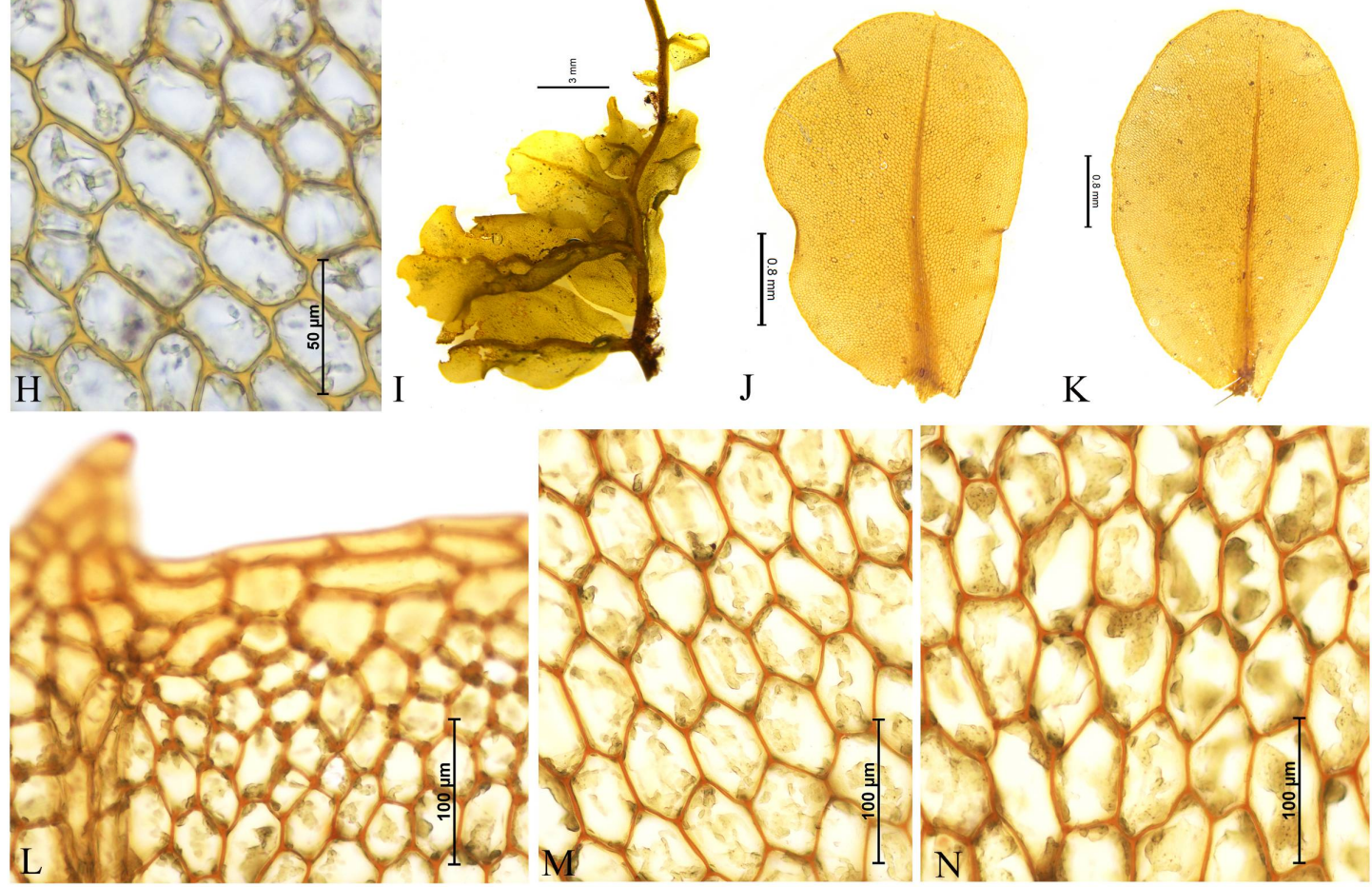

Fig. 3. (A-N). Plagiomnium rhynchophorum (Hook.) T. J. Kop . (LWG 223977B) A-H: A, B. Plant habit; C, D. Leaves; E. Leaf apex; F. Leaf apical cells; G. Leaf median cells; H. Leaf basal cells. Plagiomnium succulentum (Mitt.); T. J. Kop. (LWG 202050A) I-N: I. Plant Habit; J, K. Leaves; L. Leaf apical cells; M. Leaf median cells; N. Leaf basal cells.

6a. Leaves oblong, transversely undulated Plagiomnium confertidens*

6b. Leaves elliptic to broadly elliptical, not undulated. Plagiomnium medium

7a. Leaves with indistinct border, laminal cells much larger Plagiomnium succulentum* 7b. Leaves with distinct border, laminal cells smaller... .8

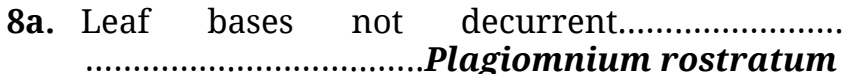

8b. Leaf bases decurrent .9

9a. Leaves with undifferentiated juxta costal cells ..Plagiomnium integrum 
9b. Leaves with differentiated juxtacostal cells.....10

10a. Plants synoicous..
Plagiomnium rhynchophorum*
rhynchophorum subsp.

10b. Plants dioecous... Plagiomnium
maximoviczii rhynchophorum subsp.

11a. Leaf margin with undifferentiated border, costa single and branched.

Pseudobryum cinclidiodes

11b. Leaf margin with differentiated border, costa single and unbranched. .12

12a. Plants green to brownish, with plagiotropic shoots....................................................13

12b. Plants reddish, erect, without plagiotropic shoots..........................................................14

13a. Leaf border strong, throughout the leaf length............................Orthomnion bryoides*

13b. Leaf border weak, restricted to base, hardly reaching till middle........Orthomnion noguchii

14a. Leaves broadly obovate, leaf apex apiculate, laminal cells large.....Rhizomnium horikawae

14b. Leaves elliptical, leaf apex not apiculate, laminal cells small..... .Rhizomnium striatulum

(*dealt in the present study)

Mnium Hedw., Spec. Musc. Frond. 188 (1801).

Mnium lycopodioides Schwaegr., Sp. Musc. Frond. Suppl. 2: 24 (1826).

\section{Description}

Plants green to reddish brown, erect, leaves closely arranged at apex, $2.5-3 \mathrm{~cm}$ long. Leaves ovate lanceolate, $2.5-3.0 \times 1.8-2.0 \mathrm{~mm}$, leaf base long decurrent, apex acute, border 2-3 layered, teeth double (paired), costa excurrent, 100-120 $\mu \mathrm{m}$ wide at base, leaf in cross section shows both dorsal and ventral stereid bands. Leaf apical cells short quadrate to isodiametric, $8-20 \times 8-20 \mu \mathrm{m}$, middle cells thick walled, corner thickenings prominent, 22.5-30 $\times$ 18.75-26.25 $\mu \mathrm{m}$ basal cells rectangular, 28-36 × 12-16 $\mu \mathrm{m}$. (Fig. 1. A-H).

Specimens examined: India, West Bengal, Darjeeling, Tonglu, ca 2500m, epiphytic, 24.04.1965, S. Chandra 202275A (LWG); Tonglu-Sandakphu, ca 3500 $\mathrm{m}$, on soil, 24.04.1965, S. Chandra 202303C, 202341B, 202360A (LWG); Sandakphu-Phalut, ca $3500 \mathrm{~m}$, epiphytic, 26.04.1965, S. Chandra 202396D, 202430F, 202432G, 202436C, 202458A (LWG); on soil, 26.04.1965, S. Chandra 202448D, 202462B, 202477H (LWG); on way to Teesta, ca $1696 \mathrm{~m}$, on stone rock, 26.09.2002, A. K. Asthana and V. Sahu 224155C (LWG); on way to Phalut from Sandakphu, ca $3636 \mathrm{~m}$, on soil covered rock, 06.11.2003, A. K. Asthana and V. Sahu 225380B, 225385A (LWG).

Distribution: India: Eastern Himalaya (Arunachal Pradesh, Sikkim, West Bengal-Darjeeling), Western Himalaya (Garhwal); Afghanistan, Bhutan, China,
Japan, Korea, Nepal, Philippines, Taiwan and Vietnam (3-7).

Other specimens studied: Musci Japonici Exsc. 67 (as Mnium laevinerve Card.), 231 (as Mnium thomsonii Schimp.) 433.

Orthomnion Wils. in Mitt., Kew J. Bot. 9: 368 (1857)

Orthomnion bryoides (Griffith) Nork., Trans. Brit. Bryol. Soc. 3: 445 (1958).

Orthotrichum bryoides Griffith, Calcutta J. Nat. Hist. 2: 486 (1842).

\section{Description}

Plants yellowish green, creeping, reddish brown rhizoids present all over the stem surface, up to $4 \mathrm{~cm}$ long. Leaves elliptical to narrowly elliptical, 3-4 $\times$ 1.5-2 mm, leaf base not decurrent, apex apiculate, border 3 layered, entire, recurved and wavy when moist, costa narrow percurrent, guide cells not visible, typical stereids lacking in the cross section of leaf. Leaf apical cells elongated hexagonal, 36-48 $\times$ 28-40 $\mu \mathrm{m}$, middle cells elongated hexagonal, thin walled, pitted, incrassate, corner thickening present but not prominent, 37-49 × 19-26 $\mu \mathrm{m}$, basal cells rectangular, 60-100 × 28-40 $\mu \mathrm{m}$ (Fig. 1. I-O).

Specimens examined: India, West Bengal, Darjeeling, Tiger Hill, ca $2500 \mathrm{~m}$, epiphytic, 19.04.1965, S. Chandra 202180A (LWG); on way to Mungpoo, ca $1757 \mathrm{~m}$, epiphytic, 26.09.2002, A. K. Asthana and V. Sahu 224129A (LWG); on way to Tonglu, ca $2489 \mathrm{~m}$, epiphytic, 02.11.2003, A. K. Asthana and V. Sahu 224346J (LWG); ca $2697 \mathrm{~m}$ epiphytic, 02.11.2003, A. K. Asthana and V. Sahu 224360D (LWG); Sirikhola, Rimbick, ca $2121 \mathrm{~m}$, on rock, 08.11.2003, A. K. Asthana and V. Sahu 225495B (LWG).

Distribution: India: Eastern Himalaya (Assam, West Bengal-Darjeeling, Meghalaya-Khasi and Jaintia Hills, Sikkim), South India; China, Laos, Myanmar, Nepal, Thailand, Vietnam. (4-7, 12).

Other specimens studied: Isotype (232004 H-SOL).

Plagiomnium T. J. Kop., Ann. Bot. Fenn. 5: 146 (1968).

Plagiomnium acutum (Lindb.) T. J. Kop., Ann. Bot. Fenn. 12: 57-58 (1975).

Mnium acutum Lindb., Acta Soc. Sci. Fenn. 10: 227 (1875).

\section{Description}

Plants yellowish green, sub erect to prostrate, upper leaves more crowded, 1.5-3 cm long. Leaves contiguous to distantly arranged, ovate spathulate, 2.5-4.0 × 1.2-1.8 mm, leaf base long decurrent, apex acute, border 3-4 layered, teeth sharp, projecting, 1-2 celled, dentate up to middle, costa reddish brown, excurrent, 120-160 $\mu \mathrm{m}$ wide at base, leaf in cross section shows only dorsal stereid band (sometimes both dorsal and ventral stereid bands present). Leaf apical cells quadrate to isodiametric, 12-24 × 8-16 $\mu \mathrm{m}$, middle cells usually isodiametric, thick walled, corner thickening absent, 15-19 × 15-23 $\mu$, basal cells rectangular, 8-28 × 12-16 $\mu \mathrm{m}$ (Fig. 2 . A-G). 
Specimens examined: India, West Bengal, Darjeeling, Lloyd Botanical Garden, ca 1818 m, on soil covered rock, 22.09.2002, A. K. Asthana and V. Sahu 223962B, 223966B, 223971B, 223977A (LWG); on way to Sandakphu, ca $3091 \mathrm{~m}$, on soil, 05.11.2003, A. K. Asthana and V. Sahu 225366A (LWG).

Distribution: India: Eastern Himalaya (West BengalDarjeeling), Western Himalaya (Kashmir); Bhutan, China, Japan, Korea, Mongolia, Nepal, Russia, Vietnam (4-7).

Other specimens studied: Musci Japonici Exsc. 68, 375 (as Mnium trichomanes), Holotype (232001 HSOL).

Plagiomnium confertidens (Lindb. \& Arnell) T. J. Kop., Ann. Bot. Fenn. 5: 146 (1968).

Astrophyllum confertidens Lindb. \& Arnell, Kongl. Svenska Vetensk. Akad. Handl. n.s. 23 (10): 17 (1890).

\section{Description}

Plants yellowish green, robust, erect, dendroid with subterranean stolons, branched, 4-7 cm long. Leaves curled when dry, oblong, elliptical to lingulate, 5-8 $\times$ 2-3 mm, leaf base long decurrent, apex acute, border 2-4 layered, teeth blunt 1-2 celled, dentate up to base, costa excurrent, 240-260 $\mu \mathrm{m}$ wide at base, leaf in cross section shows only dorsal stereid band. Leaf apical cells short quadrate to isodiametric, 8-12 ×8 $\mu \mathrm{m}$, middle cells rectangular, slightly pitted, thick walled, corner thickening prominent, 33-38 × 11-15 $\mu \mathrm{m}$, basal cells rhomboidal hexagonal 20-40 × 12-16 $\mu \mathrm{m}$ (Fig. 2. H-P).

Specimens examined: India, West Bengal, Darjeeling, Forest Rest House, ca $2000 \mathrm{~m}$, on soil, 16.04.1965, S. Chandra 202023A (LWG); TongluSandakphu, ca $2500 \mathrm{~m}$, epiphytic, 25.04.1965, S. Chandra 202322D, 202342A (LWG); SandakphuPhalut, ca $3500 \mathrm{~m}$, on soil, 26.04.1965, S. Chandra 202379C, 202400D (LWG); Kalipokhri, ca 2909 m, on soil covered rock, 05.11.2003, A. K. Asthana and V. Sahu 225357G (LWG); on way to Sandakphu, ca 3091 $\mathrm{m}$, on soil covered rock, 05.11.2003, A. K. Asthana and V. Sahu 225364C, 225376D (LWG).

Distribution: India: Eastern Himalaya (West BengalDarjeeling); Bhutan, China, Japan, Manchuria, Mongolia, Nepal, North Korea, Siberia $(4,6,7)$.

Other specimens studied: Musci Japonici Exsc. 435 (as Mnium undulatum Hedw.)

Plagiomnium rhynchophorum (Hook.) T. J. Kop., Hikobia 6: 57 (1971).

Mnium rhynchophorum Hooker, Icon. Pl. 1,tab. 20, Fig. 3 (1836).

\section{Description}

Plants yellowish green, prostrate, densely tomentose, 3-7 cm long. Leaves oblong elliptic, 3-7 × 1.8-2 mm, leaf base decurrent, apex obtuse to emarginated, border 3-4 layered, teeth blunt, 1-2 celled, not projecting, rarely absent, costa conspicuous, percurrent or mucronate at apex, 80-200 $\mu$ m wide at base, dorsal epidermis thick walled, ventral slightly thick walled, guide cells visible, leaf in cross section shows only dorsal stereid band. Leaf apical cells isodiametric to hexagonal or polygonal, $12-24 \times 8-12$ $\mu \mathrm{m}$, middle cells elongated hexagonal, 34-38 $\times 23-27$ $\mu \mathrm{m}$, thin walled, corner thickening prominent, juxtacostal cells present, basal cells rectangular, 40$68 \times 12-20 \mu \mathrm{m}$ (Fig. 3. A-H).

Specimens examined: India, West Bengal, Darjeeling, ca $2000 \mathrm{~m}$, epiphytic, 18.04.1965, S. Chandra 202075C, 202077B, 202089A (LWG); Mungpoo, ca $2000 \mathrm{~m}$, epiphytic, 18.04.1965, S. Chandra 202102A, 202105D, 202115B, 202116A, 202124C (LWG); Sandakphu-Tonglu, ca $3500 \mathrm{~m}$, epiphytic, 26.04.1965, S. Chandra 202430 D, 202454 (LWG); Lloyd Botanical Garden, ca 1818 m, on stony wall, 22.09.2002, A. K. Asthana and V. Sahu 223944B, 223977B, 223950B, 223959A (LWG); on soil covered rock, 31.10.2003, A. K. Asthana and V. Sahu 224288B, 224289A (LWG); Manebhanjang, ca $1879 \mathrm{~m}$, on soil covered rock, 01.11.2003, A. K. Asthana and V. Sahu 224311C (LWG); Singalila National Park, ca 2515 m, epiphytic, 03.11.2003, A. K. Asthana and V. Sahu 225305A (LWG); Gurdum, ca $2394 \mathrm{~m}$, on soil covered rock, 08.11.2003, A. K. Asthana and V. Sahu 225452A (LWG).

Distribution: India: Eastern Himalaya (West BengalDarjeeling, Meghalaya-Khasia Hills), South India (Nilgiri hills); Borneo, Bhutan, China, Indonesia, Nepal, North Vietnam, Myanmar, Philippines, Sabah, Sri Lanka, Taiwan, Thailand (4-8).

Plagiomnium succulentum (Mitt.) T. J. Kop., Ann. Bot. Fenn. 5: 147 (1968).

Mnium succulentum Mitt., J. Proc. Linn. Soc. Bot. Suppl. 1: 143 (1859).

\section{Description}

Plants yellowish green, prostrate, branched, 3-4 cm long. Leaves open when dry, oblong ovate to broadly elliptic, 8-10 × 6-7 mm, leaf base not decurrent, apex apiculate, border 1-2 layered, inconspicuous, entire or minutely dentate, teeth blunt, 1-2 celled, costa weak, ending below apex, 120-140 $\mu \mathrm{m}$ wide at base, leaf in cross section shows only dorsal stereid band. Leaf apical cells hexagonal, 48-88 × 40-48 $\mu \mathrm{m}$, middle cells much larger elongated hexagonal, thin walled, corner thickening absent, 101-128 × 37-41 $\mu \mathrm{m}$, basal cells rectangular, 60-88× 32-44 $\mu \mathrm{m}$ (Fig. 3.I-N).

Specimens examined: India, West Bengal, Darjeeling, on way to Manebhanjang, ca $2000 \mathrm{~m}$, epiphytic, 17.04.1965, S. Chandra 202050A (LWG).

Distribution: India: Eastern Himalaya (Assam, West Bengal-Darjeeling (Kurseong, Mungpoo), MeghalayaKhasia Hills); Bonin Islands, Bhutan, China, Japan, Nepal, North Borneo, Indonesia, Malaysia, Myanmar, Korea, North Vietnam, Philippines, Taiwan, Thailand (4-8).

Other specimens studied: Musci Japonici Exsc. 230.

\section{Discussion}

The recent study reveals the occurrence of six taxa of family Mniaceae in Darjeeling (Fig. 4) belonging to three genera Mnium, Plagiomnium and Orthomnion. Genera Mnium and Orthomnion are represented by 
single species each while Plagiomnium is represented by four species. Mnium lycopodiodes Schwaegr. is remarkably different from Orthomnion and Plagiomnium in having red colour on stem, paired tooth, two stereid bands in cross section of costa, whereas Orthomnion bryoides (Griffith) Nork. morphologically characterised by plagiotropic shoots, entire leaf margin, costa ceasing below tip or excurrent, well developed leaf border and no stereid band in cross section of costa. Plagiomnium acutum (Lindb.) T. J. Kop. comes under the section Plagiomnium T. J. Kop. of genus Plagiomnium and can be easily differentiated from all other species in
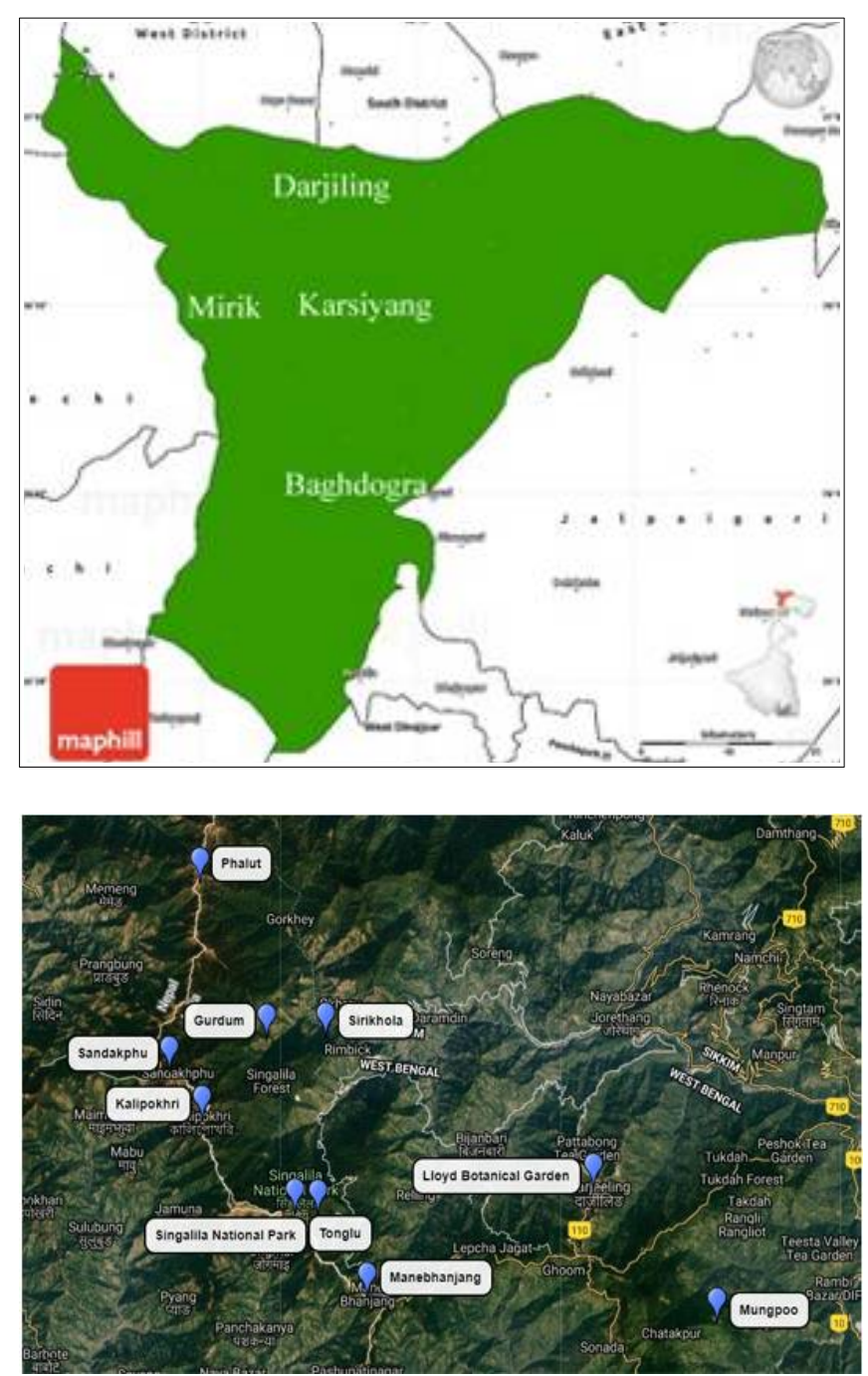

\begin{tabular}{|c|c|}
\hline \multicolumn{2}{|l|}{ Coordinates of Localities } \\
\hline Phalut & $27^{\circ} 12^{\prime} 25.398^{\prime \prime} \mathrm{N}, 88^{\circ} 0^{\prime} 56.7612^{\prime \prime} \mathrm{E}$ \\
\hline Sandakphu & $27^{\circ} 6^{\prime} 18.4752^{\prime \prime} \mathrm{N}, 88^{\circ} 0^{\prime} 5.6052^{\prime \prime} \mathrm{E}$ \\
\hline Manebhanjang & $26^{\circ} 59^{\prime} 14.19^{\prime \prime} \mathrm{N}, 88^{\circ} 7^{\prime} 18.3144^{\prime \prime} \mathrm{E}$ \\
\hline Singalila National Park & $27^{\circ} 2^{\prime} 11.8356^{\prime \prime} \mathrm{N}, 88^{\circ} 4^{\prime} 33.9312^{\prime \prime} \mathrm{E}$ \\
\hline Gurdum & $27^{\circ} 7^{\prime} 25.9284^{\prime \prime} \mathrm{N}, 88^{\circ} 3^{\prime} 29.0448^{\prime \prime} \mathrm{E}$ \\
\hline Rimbick Sirikhola & $27^{\circ} 7^{\prime} 50.6784^{\prime \prime} \mathrm{N}, 88^{\circ} 4^{\prime} 28.9884^{\prime \prime} \mathrm{E}$ \\
\hline Kalipokhari & $27^{\circ} 5^{\prime} 1.7988^{\prime \prime} \mathrm{N}, 88^{\circ} 0^{\prime} 55.1664^{\prime \prime} \mathrm{E}$ \\
\hline Tonglu & $27^{\circ} 1^{\prime} 37.0632^{\prime \prime} \mathrm{N}, 88^{\circ} 5^{\prime} 35.1456^{\prime \prime} \mathrm{E}$ \\
\hline Mungpoo & $26^{\circ} 58^{\prime} 39.7668^{\prime \prime} \mathrm{N}, 88^{\circ} 20^{\prime} 23.1828^{\prime \prime} \mathrm{E}$ \\
\hline Llyod Botanical Garden & $27^{\circ} 2^{\prime} 43.2564^{\prime \prime} \mathrm{N}, 88^{\circ} 15^{\prime} 46.0188^{\prime \prime} \mathrm{E}$ \\
\hline
\end{tabular}

Fig. 4. Map of Darjeeling showing localities of study area with their co-ordinates.

in obovate and distally toothed leaves, while Plagiomnium confertidens (Lindb. \& Arnell) T. J. Kop. belongs to section Undulata (Kindb.) T. J. Kop., having oblong leaves toothed up to base. Although Plagiomnium succulentum (Mitt.) T. J. Kop. and Plagiomnium rhynchophorum (Hook.) T. J. Kop. belong to same section Rostrata (Kindb.) T. J. Kop., but former species comprises of not decurrent leaf bases, much larger laminal cells, costa vanishing below tip whereas the latter has decurrent leaf bases, smaller laminal cells and excurrent costa.

According to earlier published literatures (4-6), all the 18 species described and enlisted by them were treated in genus Mnium Hedw. which are now placed in distinct genera with 14 species $(1,3,6,8$, 13). During the present study, it was found that some species described earlier have been synonymized or their status is changed. This work is based on the collections made from selected 10 localities of Darjeeling and specimens were deposited in CSIRNational Botanical Research Institute, Lucknow (LWG). After critical and comparative study with authentic Herbarium specimens obtained on loan from Finnish Museum of Natural History, Botanical Museum (H) and recent literature, the updated number of taxa in Darjeeling is now 15 with Plagiomnium acutum (Lindb.) T. J. Kop. as new report to eastern Himalaya.

\section{Acknowledgements}

The authors are grateful to the Director, CSIRNational Botanical Research Institute, Lucknow for encouragement and providing the facilities. Thanks are also due to Curator of Herbaria, University of Helsinki, Finland and The Hattori Botanical Laboratory, Japan (NICH) for sending the authentic specimens (on loan) for our study.

\section{Competing interest}

Authors do not have any conflict of interest to declare.

\section{Authors' contribution}

The first and second authors identified the specimens and prepared the manuscript, photo plates and map of the study area. The second and third author carried out field explorations to Darjeeling and neighbouring areas and collected the plant specimens. The third and corresponding authors finalized and investigated all the details, checked the manuscript and submitted for publication.

\section{References}

1. Koponen T. Generic revision of Mniaceae Mitt. (Bryophyta). Ann Bot Fenn. 1968; 5:117-50

2. Koponen T. A synopsis of Mniaceae (Bryophyta) X. Melanesian taxa, with a world list of the taxa in Mniaceae. Acta Bryolichenol Asiat. 2017; 7: 205-27 
3. Koponen T. On the hypothesis of dioicous-monoicous species pairs in the Mniaceae (Bryophyta); morphology, sexual condition and distiribution. Acta Mus Siles Sci Natur. 2019;68:67-81

4. Gangulee HC. Mosses of Eastern India and adjacent regions. Vol II. Books and Allied Pvt. Ltd., Kolkata; 1974

5. Chopra RS. Taxonomy of Indian Mosses. C.S.I.R. Publication, New Delhi; 1975

6. Koponen T. A synopsis of Mniaceae (Bryophyta). VI. Southeast Asian taxa. Acta Bot Fenn. 1981;117:1-34

7. Lal J. A checklist of Indian Mosses. Dehra Dun, India; 2005

8. Koponen T. The East Asiatic species of Plagiomnium sect. Rostrata (Bryophyta). Acta Bot Fenn. 1972;97:1-29
9. Koponen T. A preliminary report on the Mniaceae in Japan II. Hikobia. 1974; 7(1-2):1-20

10. Koponen T. A synopsis of Mniaceae (Bryophyta). IV. Taxa in Europe, Macronesia, NW Africa and the Near East. Ann Bot Fenn. 1980;17(2):125-62

11. Koponen T. Bryophyte flora of Hunan Province, China. 18 Mniaceae subfam. Mnioideae (Musci). Acta Bryolichenol Asiat. 2014;5:39-72

12. Koponen T. A synopsis of Mniaceae (Bryophyta). II. Orthomnion. Ann Bot Fenn. 1980;17(1):35-55

13. Koponen T. A synopsis of Mniaceae (Bryophyta). IX. Taxonomy, habitats, substrates and ranges of the species of Rhizomnium. Acta Bryolichenol Asiat. 2014;5:85-111 